\title{
Research the Systems Architecture and Technology of Wisdom Community Based on the Internet of Things
}

\author{
ZHU Shun-bing ${ }^{1,2, a}$, DU Chun-quan ${ }^{2}$ and NIU Miao-miao ${ }^{2}$ \\ ${ }^{1}$ Jiangsu Key Laboratory of Urban and Industrial Safety, Nanjing University of Technology, Nanjing \\ 210009,China \\ ${ }^{2}$ School of Urban Construction and Safety Engineering, Nanjing University of Technology, Nanjing \\ 210009, China \\ a13913399658@139.com
}

Keywords: Internet of Things (IOT), wisdom community, smart home, architecture, cloud platform, function

\begin{abstract}
The wisdom community is the basic unit of the smart city, is a set of urban management, public services, social services, residents' autonomy and mutual aid services in one of the new technology applications. This article analyzes the current situation and existing problems of the wisdom community, then described the Internet of Things architecture, equipment features, community cloud computing platform and structure, the last detailed analysis of the wisdom community features and community network video intercom, home security, appliance control, noncontact card access control, card consumption management, community security, community eservice technology and other technical content and features.
\end{abstract}

\section{Introduction}

With the rapid development of Internet of Things (IOT), cloud computing, IT has an important significance for economic and social change, developed cities have the construction of the smart city as an important means for strengthen urban competitiveness, promote industrial upgrading and structural adjustment, enhance the city's innovation capacity and sustainable development. The wisdom community is the basic unit of the smart city, is a set of urban management, public services, social services, residents' autonomy and mutual aid services in one of the new technology applications. It is based on the Internet, rely on the use of the IOT technology, the smart home systems, community's material systems and services associated together, so that the community managers, users, and a variety of intelligent systems to the formation of various forms of information exchange, in order to achieve a more convenient and efficient management, giving the users more comfortable digital life experience. The implementation of the wisdom community, reinforce the smart city concept, significance and role in society.

Wisdom community is on the basis of the smart city, more specific proposed, but smart city proposed a very short time, due to the following problems: lack of unified planning and standards, bull management, redundant construction and lack of suitable business models. So the wisdom community building field is fragmented and the application level is not high, promote the overall trend has not formed on the surface. There is no effective business model and profit model, to be studied to solve these problems.

\section{IOT Architecture of the System of Wisdom Community}

System Architecture. According to the characteristics of the wisdom community of Things, it can be divided into a device layer, application layer and value-added service. Fig.1 shows the wisdom community systems of Things architecture. 


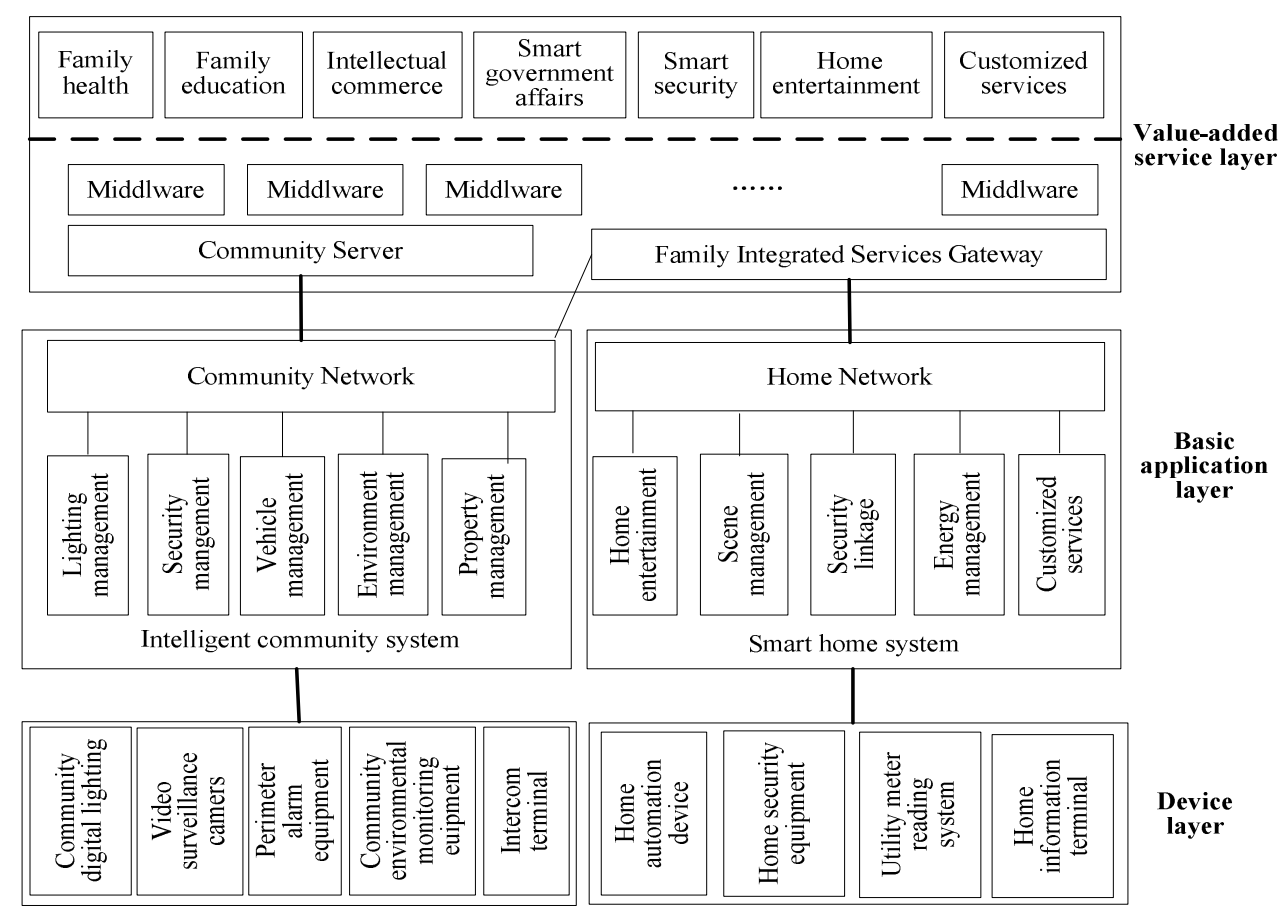

Fig. 1 Internet of Things architecture of the system of wisdom community

Device Layer. Device layer is composed of all electrical equipments within the community and smart home system electrical equipments, including digital lighting, camera surveillance equipment, perimeter alarm equipment, parking equipment, video intercom terminal equipment, home automation equipment, home security equipment, household energy consumption metering devices and home intelligent terminal.

The device layer has the following characteristics: (1) digital information interfaces, via the bus (or network) for two-way communication; (2) the IOT interface (RFID scalable perception interface); (3) provide data services for the application layer.

Application Layer. The application layer is divided into two parts: the wisdom community systems and smart home systems. The wisdom community systems completed the task of the wisdom community, including lighting management, security management, parking management, district environmental management and property management. The smart home system is a functional unit for every household, including home entertainment, scene management, security linkage, energy management and customized services. Application layer device (software) has the following characteristics:(1)digital information interface, via the bus(or network) for two-way communication; (2) the IOT interface, such as RFID scalable perception interface; (3) the function of superior service, provide the necessary interfaces and services for the value-added service layer.

Value-added Service Layer. Value-added services interface provided by the Community Server. Value-added services providers can be the property also can be a specialized external provider.

Value-added service layer has the following characteristics: (1) The service is connected through the middleware and Community Server (or family business gateway), should be applied to a variety of hardware and software platforms. (2) Value-added service is an extension of the basic application, satisfied the specific service requirements of the users of certain services, shall be based on the basic application; (3) allows the wisdom community to better support the smart city's building, more conducive to the wisdom community to become the necessary functional units of the smart city.

Wisdom Community Cloud System. Build cloud computing platform can both use for community computing, data storage and processing, can effectively raise the level of wisdom community life, focus on the various subsystems to provide the ability to support, and data sources. Cloud computing platform mainly for the intelligent home, the wisdom of old age, smart security, smart business to provide data services, the structure shown in Fig. 2. 


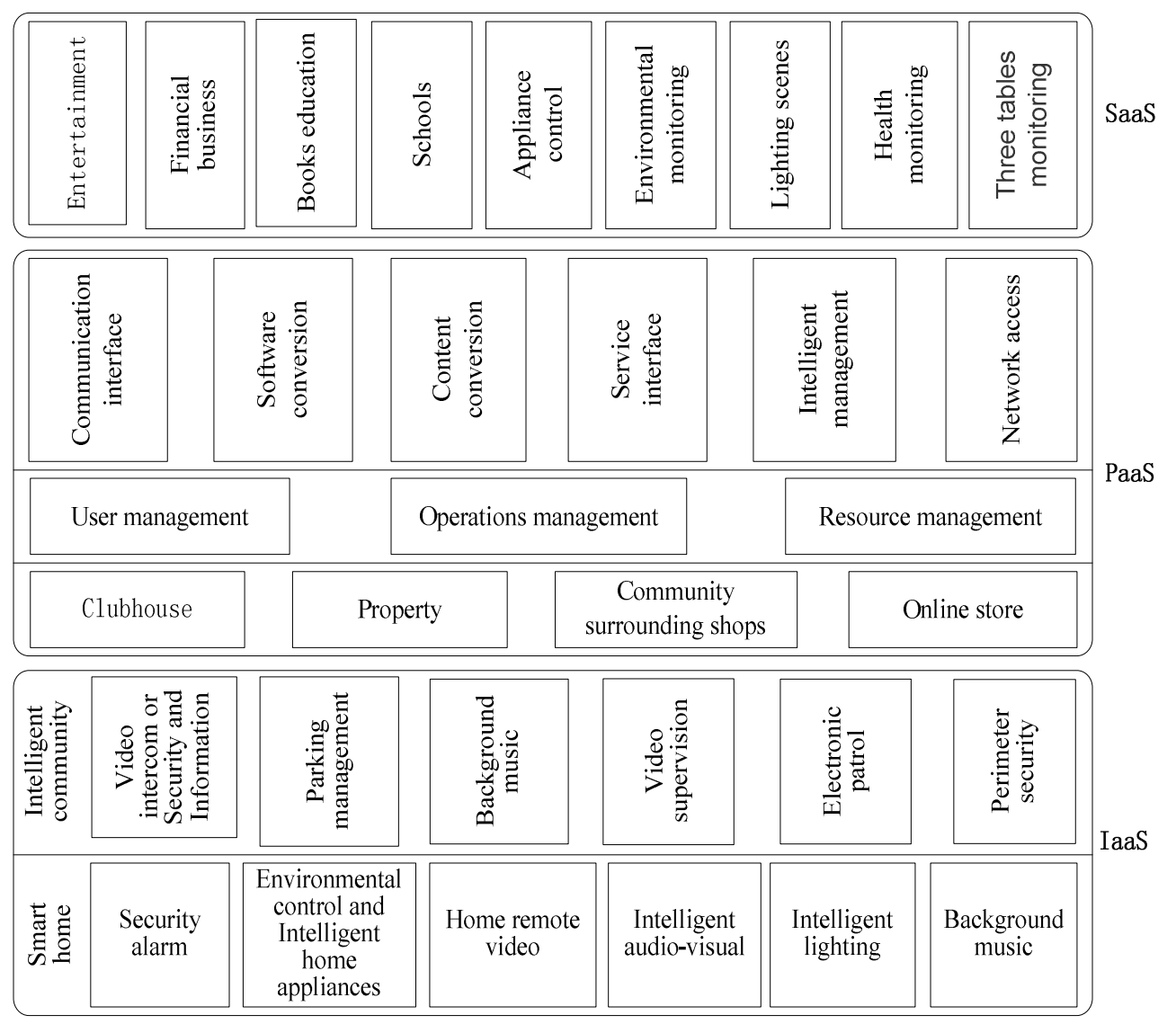

Fig. 2 The architecture graph of community cloud system

Community cloud system is mainly composed of three parts, divided into three levels according to the standard architecture of cloud computing, the bottom layer ("laaS" infrastructure and services), including the family of intelligent and community intelligence, which is the basis of the cloud community, assumed the underlying network, infrastructure and intelligent systems. The top level ("SaaS" software and services) reflect a variety of services, to provide users with a variety of life, entertainment, education, security services. The middle layer ("PaaS" platforms and services) is the core of the system, to undertake various services on the Internet, and network resources of the various services rendered to the community residents.

\section{Functions and Technical Characteristics of the Wisdom Community}

Wisdom community consists of two parts, one part is the smart home, the family of intelligent, the second part is public areas of intelligent, system topology shown in Fig. 3. Smart home join in the wisdom of community, digital, user-friendly management can be achieved, and then through the Internet portal as the entrance, enabling users to interact the residential intelligent management systems and smart home systems through the terminals .

Smart Home. The systems include security systems, remote monitoring, smart home appliances, video intercom, scene mode, intelligent audio-visual and other subsystems, the users can easily manage the remote terminal management systems.

Security System. The system ensures that the user's life and property security, consists of home alarm hosts and a variety of front-end detectors. The front-end detectors can be divided into a door sensor, magnetic window, gas detectors, smoke detectors, infrared sensors, emergency buttons, et al. If illegal invasion would trigger the corresponding detector, home alarm system will immediately sent the alarm signal to the community center or user-specified phone, so that security personnel quickly out of the police, while community center alarm host will be recorded these information for inspection. 


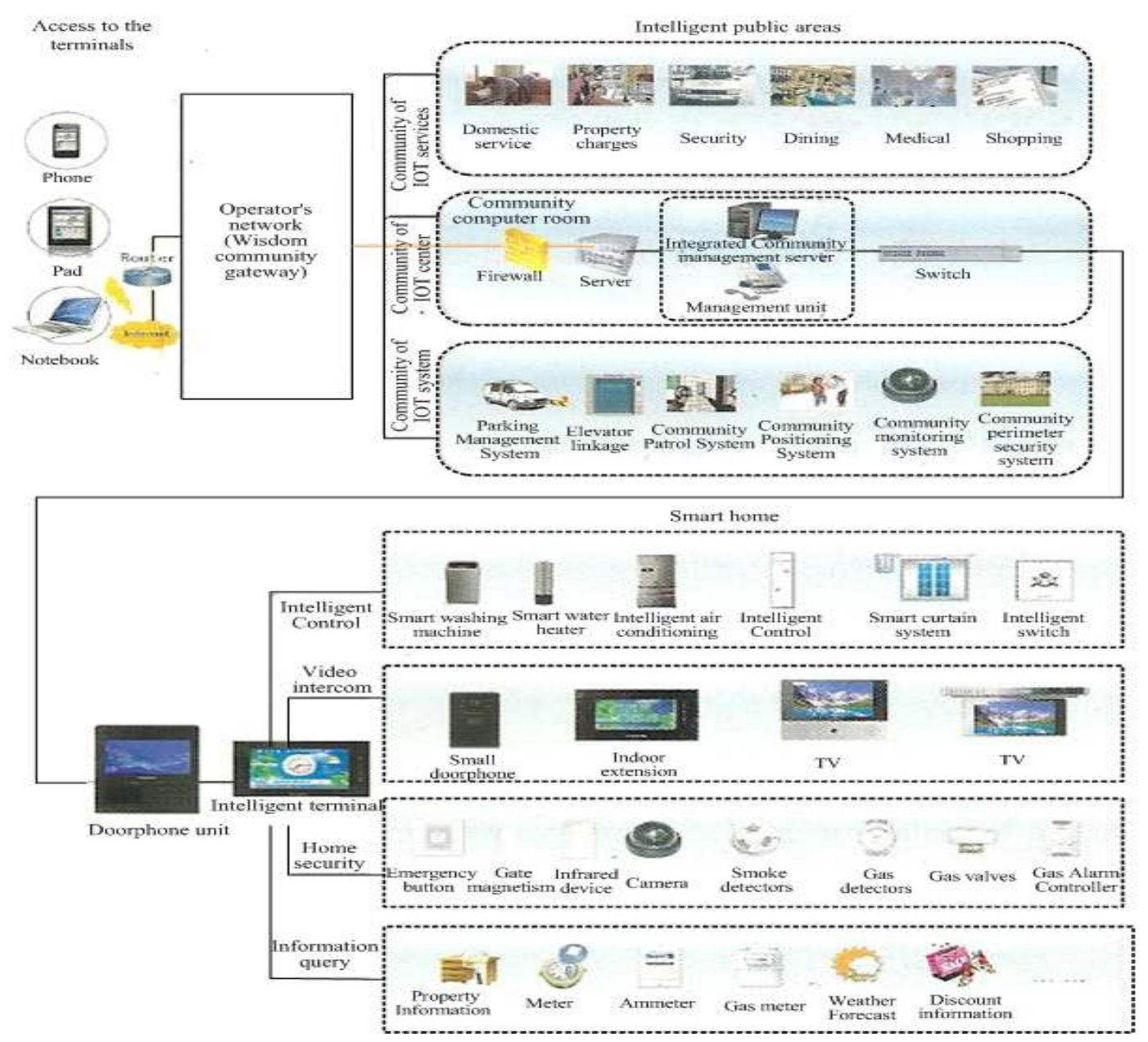

Fig. 3 The topology of the wisdom community system

Remote Monitoring System. Users can take advantage of the $3 \mathrm{G}$ handsets and the Internet, browse the video images anytime and anywhere, at the same time the system supports multiplatforms access of the WEB site, the client and the phone log, with video browsing capabilities, can meet the users image split-screen view, the history of video viewing, photo capture and PTZ control.

Smart Home Appliances. The system through the family intelligent terminals and intelligent remote control of the household appliances, can control all electrical equipments in the home network, including lamps, electric curtain/roller blinds, general electrical appliances, high-power electrical appliances, infrared electrical appliances(such as TV, air conditioning, VCD, audio, et al.) and so on.

Video Intercom System. Owners or visitors can call any indoor extensions within community through the door machine, also can with the owners of indoor do video intercom and unlock operation. If owners were not at home, you can leave pictures and messages. When owners came home, can viewed day visitors, and saw the visitor's voice and video message. Between the owners can do the household video intercom, the entire system can be multi-way video intercom does not interfere with each other. Door machine or indoor host can call the property management personnel do video intercom.

Scene Model. Set the scene panel, you can control the whole house within the designated area lighting, air conditioning/heating system, audio and video systems, et al.

The system also can be set to a variety of scenes (such as light shade combination, with a combination of audio equipment, multi-mode, based on the habits of the owner's to do the postdesign). Set the smart background music panel, can be turned on and off the background music, select the tracks and new broadcasts at the same time, call home or call nanny service. 
Intelligent Audio and Video System. The whole house background music sharing so that each room can listen to music independently, switch audio, adjust the volume, and without disturbing each other. Whole house audio and video sharing system set the background music and videosharing in one.

Wisdom Community. The community center room as the hub of the system, combined the intelligent home households, community digitized service, property digitized manage, community intelligent management, to achieve: (1) information unicom between the tenants (2) households enjoy the fast services: residential electronic shopping, housekeeping, automatic toll collection (3) community management automation.

Community Intelligent Service System. (1) Smart card system. By a non-contact identification card can achieve access control, parking access, low consumption and a variety of different functions of the wisdom of management.

The access control system installed in each apartment unit doors, important equipment rooms, elevator car, car park entrances and flow entrances, et al. The cell door use one-way card reader, the main engine room and the main entrance with two-way card reader. Management approach with centralized management, regional controller via RS485bus data aggregated to the two engine room, through the TCP/IP transmission to the monitoring center engine room.

(2) Card consumption management. Based on the broadband network connectivity of all households, community parking/access control system IC card as the carrier, establishment of a unified operational service center, combined with the wisdom community and call center, to coordinate the back-office operations, front desk departments and collaborative businesses, providing owners with a comprehensive, package of services, including community property, selfvalue-added services, the association of the agency business, community and surrounding joining in the membership-based business services. The card owner can use the Internet, telephone, call center and a variety of ways, through the scheduling of the community card operations center on the internal, external commercial organizations, to obtain various types of value-added services and extension services, as detailed in Fig. 4. The owners with a card can enjoy all the community services, property management companies can take advantage of this system fully operational services to the households, access to value-added business profits.

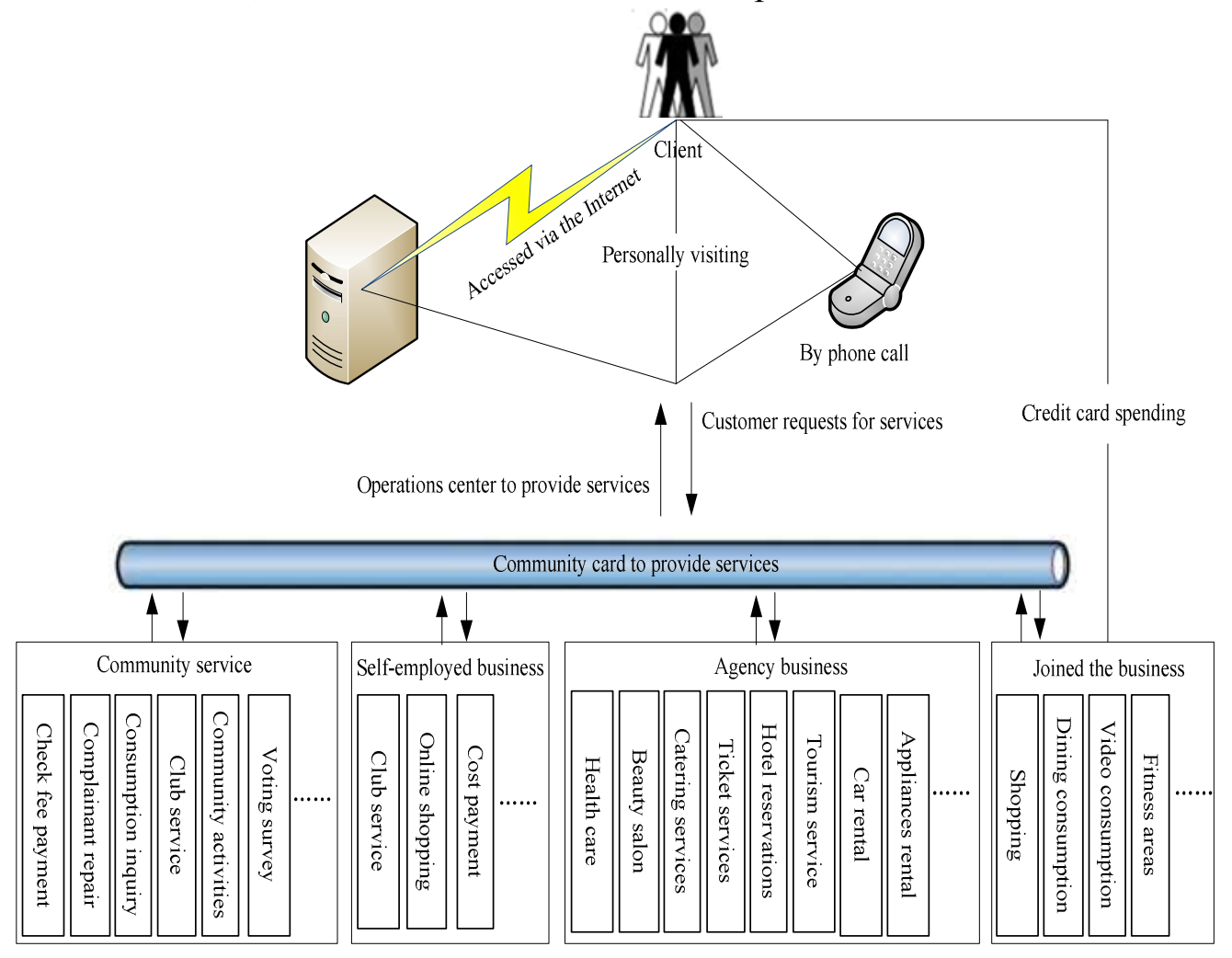

Fig. 4 The system program of wisdom community one-card 
Community Intelligent Management System. (1) Video surveillance system. Systems are arranged in the entrances of each apartment unit, the main roads within the community, underground parking, important equipment rooms, office service areas in public buildings, as well as some important areas to be fortified.

(2) Electronic patrol system. Off-line electronic patrol system has characteristics of no special wiring and easy expansion.

(3) Parking management system. The system consists of several aspects of vehicle management, parking transportation planning, pedestrian-induced. All entrances are network management. The charging method using Mifare cards is divided into fixed, temporary, and other billing methods.

(4) Emergent rescue system and home security system. System manages the gas, door magnet, infrared and emergency buttons and other sensor alarm signals, indoor host via a dedicated data bus sent the alarm signals to the management center, the management computers install the management software to set the visitor intercom, able to bring up the electronic maps in the alarm to identify the specific alarm household unit building and unit number, the staff on duty are more intuitive, easier to manage.

(5) Burglar alarm system. The system used for the prevention of the key parts, import and export, complete the fortified zone automatic or manual, disarm, and deployment point for group management. Districts alarm signals access this area of security equipment alarm host, through the partition alarm host to the integrated management and monitoring command center. At the same time, set in throughout the detectors have the linkage function with the video surveillance and access control.

(6) Remote meter reading metering system. Remote meter reading metering system focuses on the management of the household water, electricity, gas and other measurements.

(7) Construction equipment management system. The system includes water supply and drainage systems, power distribution systems, lighting in public areas, elevator systems, et al. Monitoring center through the control network and distribution in the accused device near the site controller to communicate, the site staff can use the display panel and operating panel to operate or monitor device.

(8) Community e-service system. (1)Basic services: weather forecast, community notification, peripheral information, newsletters, reminder collar, electronic billing and convenience. (2) Advertising services. Sensors are settled on the door machine. Once anybody is approach to the sensors the door machine broadcast advertising or community notification (custom protocol). The door unit information services page publish similar ads (browser mode). The indoor unit pops ads mandatory (custom protocol). (3) Neighboring merchants online sales. (4)Community clubs, service facilities reservation, include: clubhouse, sports center, chess room, swimming pool, reading room, health center. (5)Houses agency and housekeeping. (6) Security services. The automatic messages alarm and automatically forwarded to a security company or 110. (7)Kindergarten video monitoring service. Permissions restrict specified by the camera. (8)Health, elderly care services (9)Finance, stock information, online music, video.

\section{Summary}

The wisdom community of Things architecture is the necessary conditions for wisdom community toward the large integrated application environment, integrated model promote the wisdom of the community cloud computing services development. Community contact with the masses most closely, wisdom community building, neither a simple emphasis on infrastructure, can not be overly obsessed with the advanced technology, but should be based on real-life, demand-driven, using a reliable, cost-effective information technology, available to the masses a convenient, fast, attentive service and help.

Community in fact is the city last mile/100 meters/ 10 meters of grass-roots units, also is the city fully reflects the people-oriented. After more than ten years of development, China has formed a relatively complete, self-development wisdom community industry chain. To develop the wisdom 
community, must first implement the development of the wisdom community industry chain, in particular, the development of independent innovation products and systems in line with national conditions.

The wisdom community building is a systematic project, which requires multi-stakeholder involvement. In order to avoid duplication, reduce the workload of the community and to improve the accuracy of the data, should be unified the community data collection methods, draw up the building norms and standards.

Jiangsu Province Industry and Information Technology Transformation and Upgrading of A Special Guide Capital Project (2011 C-class provincial 06)

\section{References}

[1] Li Jian, Zhang Chunmei, Smart city and its evaluation and assessment methods to study, J. Telecommunications Network Technology. 1 (2012) 1-5.

[2] Zhang Peng, Wang Yibin, Chen Yumei, Li Ruosi, To build intellectual community based on the urban and rural integrated information service platform, J. China Management Informationization. 15 (2012) 83-84.

[3] Li Deren, From the digital city to the smart city, J. Information of China Construction. 23 (2011) 10-12.

[4] Zhang Qingsheng, Li Mengru, Haier cloud community: a life enjoy no domain, J. House Industry. 1 (2011) 83-88.

[5] Guo Sihong, Intelligent system of residential community for smarter city, J. Modern Architecture Electric. 2 (2011) 1-4.

[6] Gao Yunsheng, Real estate new business model-cloud community, J. House Industry. 1 (2012) 26-29.

[7] Li Jia, Wu Yi-rong, Construction scheme of digital community based on Internet of Things, J. Computer Engineering. 37 (2011) 262-263.

[8] Xu Heyuan, Li Jian, Qu Zhenhua, Luo Song, Tang Libo, Researches on the meaning and application service system of smart city, J. Telecommunications Network Technology. 9 (2011) 14-18.

[9] Li Xianyi, Deng Xiaoyu, Smart city evaluation index system, J. Telecommunications Network Technology. 10 (2011) 43-47.

[10]Wang Bin, Introduction of 《The Internet of Things application guidelines of digital community 》, J. Housing Science. 179 (2011) 88-91. 